Mini-Review for Current Medicinal Chemistry

Reference\#: BMS-CMC-2020-289

\title{
Functional Role of Glycosphingolipids in Cancer
}

Changping Zheng ${ }^{\text {a }}$, Marco Terreni ${ }^{c}$, Matthieu Sollogoub ${ }^{a}$, Yongmin Zhang a,b,*

${ }^{a}$ Sorbonne Université, CNRS, Institut Parisien de Chimie Moléculaire (UMR 8232), 4 Place Jussieu, 75005 Paris, France; ${ }^{b}$ Key Laboratory of Tropical Medicinal Resource Chemistry of Ministry of Education, College of Chemistry \& Chemical Engineering, Hainan Normal University, Haikou, 571158, China; ${ }^{c}$ Drug Sciences Department, University of Pavia, Viale Taramelli 12, 27100 Pavia, Italy.

*Corresponding authorＥ-mail: yongmin.zhang@upmc.fr 
Abstract: Glycosphingolipids (GSLs) are ubiquitous components on animal cell membranes, and exposed on the outer surface. Various studies have demonstrated that they play key roles in cells proliferation, adhesion, motility and differentiation. Usually, the specific types of GSLs are expressed more highly in tumors than in normal tissues, which are known as tumor-associated antigens. It has been revealed that most tumor cells show altered GSLs patterns on their surface, abnormal GSLs signaling and biosynthesis, which together play a major role in tumor development. Tumor-associated GSL antigens have been used in the development of antitumor vaccines. It is no doubt that GSLs play a crucial role in tumor progression and would be a promising target for cancer treatment.

Keywords: glycosphingolipids, tumor-associated antigens, GSL signaling, carbohydrates, medicinal chemistry, cancer. 


\section{Introduction}

Glycosphinolipids (GSLs) consist of a hydrophobic ceramide (Cer) moiety and a glycosidically bound carbohydrate moiety [1]. In general, GSLs can be categorized into two groups: neutral and negatively charged (sialic acid or sulfate group) GSLs, and the four most common ones are globo-, lacto-, neolacto- and ganglio- series [2]. The typical examples of GSLs are listed in Figure 1.

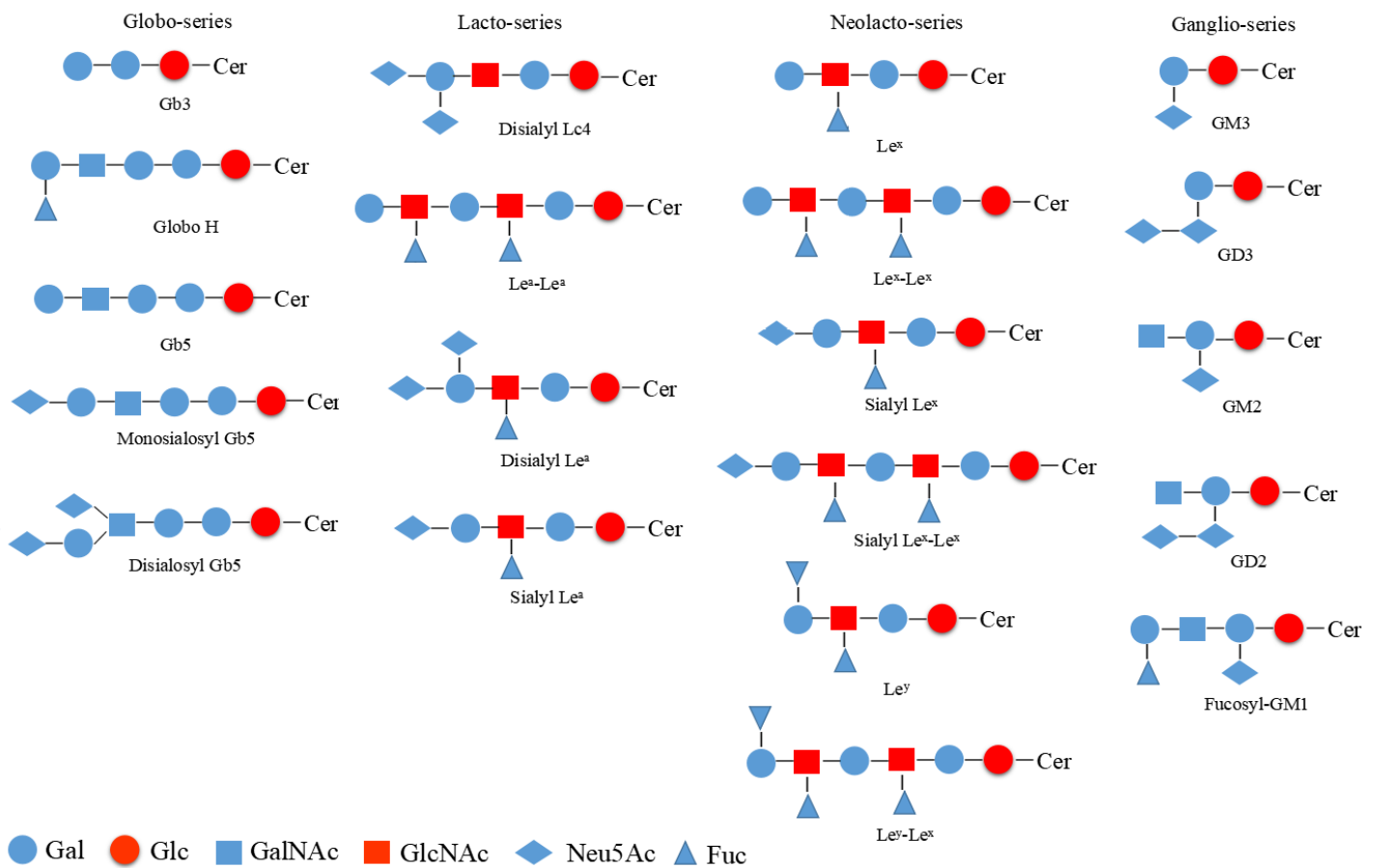

Figure 1. Structure of typical GSLs

More and more evidences showed that GSLs have significant effects on tumor development and progression, which can modulate the cancer cells proliferation, adhesion, invasion and apoptosis [3-5]. It is well known that GSLs are tumor-associated carbohydrate antigens (TACAs) on some types of cancers. They are present in both normal tissues and cancer cells, but highly expressed in tumor cells, and changes in GSLs synthesis occur frequently in cancers, especially the aberrant 
sialylation of GSLs [6]. The expression of GSLs on some human cancers is shown in Table 1. The abnormal expression of GSLs on different cancers can provide the valuable sight to develop the new strategies for cancer treatment. This mini-review will present the role of GSLs in some common happened cancers.

Table 1. Expression of GSLs on some types of cancers

\begin{tabular}{|c|c|}
\hline GSLs & Tumor types \\
\hline GM3 & melanoma, medulloblastoma, superficial bladder cancer \\
\hline De-N-acetyl GM3 & melanoma \\
\hline Neu5Gc GM3 & melanoma, colon and breast cancers, retinoblastoma \\
\hline 9-O-acetyl GM3 & melanoma, glioblastoma \\
\hline GM2 & melanoma, neuroblastoma, medulloblastoma \\
\hline GM1 & small cell lung carcinoma, renal cell carcinoma \\
\hline Fucosyl-GM1 & small cell lung carcinoma \\
\hline 9-O-acetyl GD3 & melanoma, neuroblastoma, breast cancer \\
\hline GD2 & melanoma, neuroblastoma, retinoblastoma \\
\hline 9-O-acetyl GD2 & ovarian cancer, neuroblastoma, small cell lung cancer \\
\hline $\mathrm{Gb3}$ & ovarian cancer, burkitt lymphoma \\
\hline Globo $\mathrm{H}$ & ovarian and breast cancers, teratocarcinoma \\
\hline Disialosyl galactosyl globoside & renal cell carcinoma \\
\hline Gb5 & teratocarcinoma \\
\hline Monosialyl Gb5 & renal cell carcinoma \\
\hline Disialyl Gb5 & renal cell carcinoma \\
\hline Disialyl Lc4 & colon cancer \\
\hline $\mathrm{Le}^{\mathrm{a}}-\mathrm{Le}^{\mathrm{a}}$ & gastric, colon, bladder and breast cancers, Hodgkin's lymphoma \\
\hline Sialyl Le $\mathrm{Le}^{\mathrm{a}}$ & colon, pancreatic and gastric cancers \\
\hline Disialyl Le ${ }^{\mathrm{a}}$ & colon cancer \\
\hline $\operatorname{Le}^{\mathrm{x}}$ & gastric, breast and colorectal cancers \\
\hline $\mathrm{Le}^{\mathrm{x}}-\mathrm{Le}^{\mathrm{x}}$ & colon, gastric, breast, lung and liver cancers \\
\hline
\end{tabular}




\begin{tabular}{c|c} 
Sialyl Le & colon and bladder cancers, T cell leukemia \\
Sialyl Le ${ }^{\mathrm{x}}-\mathrm{Le}^{\mathrm{x}}$ & colon, gastric and lung cancers \\
$\mathrm{Le}^{\mathrm{y}}$ & colon, gastrointestinal, colorectal, lung and breast cancers \\
Le $^{\mathrm{y}}-\mathrm{Le}^{\mathrm{x}}$ & colon, liver, lung, colorectal and pancreatic cancers \\
\hline
\end{tabular}

\section{Bladder cancer}

Some studies reported that exogenous ganglioside GM3 has significant antitumor effects on bladder cancer cells. For example, in cell lines YTS-1, T24, T5637 and KK47, increasing GM3 can reduce cells proliferation, adhesion and epidermal growth factor receptor (EGFR) phosphorylation [7]. The antitumor effect of exogenous GM3 has also been revealed in the murine bladder cancer cell line MBT-2, and apoptosis was induced when cells were transfected with GM3 synthase cDNA [8]. Other studies showed that adding GM3 to the human bladder cancer cell lines KK47 and T24, the invasive property was decreased [9]. Exogenous GM3 can inhibit bladder tumor cells invasion which indicated that GM3 expression can reflect the invasion potential to some extent.

Moreover, some data demonstrated that a specific microdomain ("Glycosynapse 3") can control phenotypic conversion and reversion of bladder cancer cells through GM3-mediated interaction of $\alpha 3 \beta 1$ integrin with CD9 [10]. Through studying the functions of GM2 and GM3 in cell motility and growth in bladder cancer, it has been showed that GM2/GM3 complex affixed on silica nanospheres strongly inhibits cell motility through CD82/cMet-mediated pathway [11,12].

Now it is known that up to $90 \%$ of bladder tumors express Le ${ }^{\mathrm{X}}$ antigen [13]. In particular, the sialyl $\mathrm{Le}^{\mathrm{X}}$ antigen has closely associated with the invasive and metastatic potential of bladder cancer, and the importance of sialyl $\mathrm{Le}^{\mathrm{X}}$ as a marker of invasive and metastatic potential was noted [14]. 


\section{Renal cancer}

It was reported that after implantation of renal tumor cells into BALB/c mice, tumor volume was increased when LacCer was upregulation. However, tumor volume was greatly reduced by treatment with D-PDMP, an inhibitor of GlcCer synthase (GCS) and LacCer synthase [15]. In addition, the expression of GM3 in renal cancer patients was higher than in healthy controls [16]. The high disialosyl globopentaosylceramide (DSGb5) expression levels exhibit stronger migration potential in renal cancer cells [17]. The results suggest that certain GSLs play a very complex role during the development and progression in renal tumor.

Next, the disialosyl-galactosylgloboside was also found to be highly expressed in human renal cancer [18], and the expression of disialosyl-galactosylgloboside is associated with the potential of renal tumor cells metastasizing to the lung. Further study showed that expressing high levels of disialosyl-galactosylgloboside adhere strongly to perialveolar lung tissue sections, and it can be inhibited by a mAb (RMZ), which specifically direct to disialosyt-galactosylgloboside. It is resumed that a specific receptor may be present in lung tissue, and specific interaction between receptor and disialosyl-galacrosytgloboside can mediate the metastasis of renal cancer to the lung $[19,20]$.

\section{Colon cancer}

GSLs and related enzymes abnormally expressed in colon cancer have been studied. Studies on colon cancer patients showed that Gb3 expression is increased, which is the receptor of Shiga toxin and binds to the B-subunit or its derivatives $[21,22]$. The expression of Gb3 strongly correlates with the metastatic potential, and high levels of its expression and a migratory phenotype were found based on three colon cancer cell lines established from metastatic human colon cancers [23]. Furthermore, in human colon adenocarcinoma SW620 cells, the antitumor effect of anti-epithelial cell adhesion molecule (EpCAM) mAb can be enhanced strongly by 
gangliosides GD1a and GM1 expression [24]. The tests also showed that $\alpha$-GalCer has the therapeutic effect on colorectal cancer. In AOM/DSS mice, when treated with $\alpha$-GalCer, the number of colorectal tumors was significantly reduced [25].

It has been reported that in human colorectal carcinoma HCT116 cells, Gb4 can enhance activation of EGFR induced MAPK/ERK signaling by direct interaction with EGFR [26]. Another study demonstrated a better cytotoxicity of GM3 analogues against colorectal carcinoma HCT116 cells than HaCaT normal cells, and the cytotoxicity of these derivatives was thought to be caused by their inhibition on activity of various growth factor receptor (GFR)-associated tyrosine kinases [27]. Besides, it is well known that cisplatin is used for colorectal cancer therapy, and in HCT116 cells, oxidative apoptosis mediated by GM3 was shown to be associated with cisplatin-induced apoptosis [28].

NEU3 that is a human plasma membrane-associated sialidase, is upregulated in colorectal tumor. NEU3 plays an important role in malignancy [29], and is also involved in inflammation-dependent cancer development [30]. The EGFR activation is enhanced by NEU3 through desialylation with no effects on EGFR mRNA or protein expression [31]. Furthermore, silencing NEU3 results in significant decrease in clonogenicity along with down-regulation of stemness and Wnt-related genes in HT-29 and HCT116 cell lines [32].

\section{Melanoma}

Ganglioside GM2 antibodies in melanoma patients associating with improved disease-free and overall survival were observed [33]. It has been confirmed that GM2 is the most immunogenic ganglioside in the melanoma [34]. Besides, ganglioside GD2 can block programmed death receptor 1 (PD-1), and PD-1 blockade enhanced

both chimeric antigen receptor (CAR) T-cells survival and promoted killing of PD-L1(+) tumor cell line [35]. In HT-144 cell line, the ganglioside GM4, GM3 and GD3, purified from human melanomas, can inhibit the phenotypic and functional 
differentiation of monocyte-derived dendritic cells induced by CD154. Furthermore, increasing GM3 and GD3 not only induce strong monocyte-derived dendritic cells apoptosis, but also decrease interleukin-2 (IL-2) and interleukin-10 (IL-10) concentration [36].

In human melanomas, GD3 is the predominant ganglioside component $[37,38]$. Activation of natural killer T (NKT) cells produces cytokines to influence immune responses against cancers, and GD3 can induce NKT cells response in melanoma. Moreover, it has been confirmed that GD3 can enhance malignant properties of melanomas, and the mechanism is shown in Figure 2. The molecules involved in GD3-mediated signaling pathways include focal adhesion kinase (FAK), p130Cas and paxillin. RNAi can block p130Cas and paxillin, which can obviously suppress melanoma growth [39]. In $\mathrm{GD}^{+}$, not GD3-, human melanoma N1 cells, the proliferation and apoptosis resistance were enhanced through hepatocyte growth factor (HGF) or adhesion to collagen type I via MAPK and Akt signaling pathways. Increased GD3 expression can promote melanoma cells adhesion to surrounding tissues and susceptibility to HGF presenting in the tumor microenvironment [40,41]. Other results further showed that the melanoma malignancy is enhanced by GD3 through g-secretase recruiting to rafts and facilitating efficient cleavage of neogenin [42]. It has also been found that 9-O-acetylated GD3 presents in malignant melanomas [43].

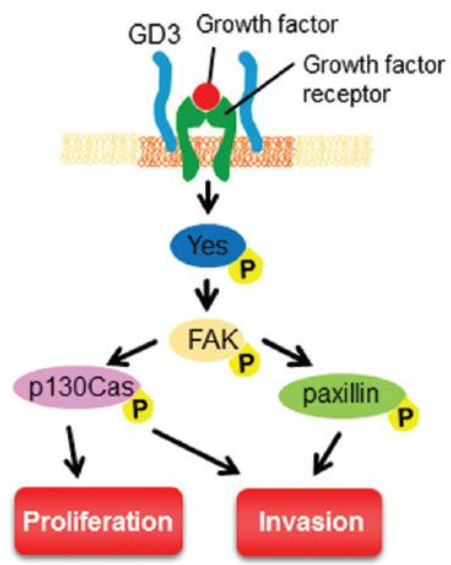


Figure 2. Mechanism for malignancy enhancement by GD3 in melanomas. GD3 can activate FAK, p130Cas and paxillin, thus promoting cells proliferation and invasion.

The expression of GM3 on the invasive and metastatic properties in four mouse melanoma B16 variants has been studied. In B16, the GM3 expression is the highest, and it is the most invasive and metastatic type, followed by F10, F1 and WA4. It has been reported that GM3 can be regarded as an adhesion molecule for another two GSLs, gangliotriaosylceramide (Gg3Cer) and LacCer. The metastasis of $\mathrm{B} 16$ is triggered through the recognition of GM3 by Gg3Cer or LacCer on endothelial cells, which is a type of GSL-GSL interaction, and the interaction results in signals conduction [44,45]. In addition, 5-N-deacetylation of GM3 has already been confirmed to specially express in metastatic melanomas, but not in normal tissues or in the most of primary melanomas or benign nevi [46]. Further results demonstrated that 5-N-deacetylation of GM3 can stimulate both cells migration and invasion through increasing the expression and activation of urokinase-like plasminogen activator ( $u \mathrm{PA})$ and matrixmetalloproteinase-2 [47]. Another study showed that in melanoma B16 cells "GSL signaling domains" are present [48], and the related results indicated that clustered GSLs associated with c-Src, Rho A and FAK are involved in GSL-dependent cells adhesion coupled with signal transducers activation [49,50]. For example, GM3 clustering forms a "glycosignaling domain" (GSD), and in this case, the antigenicity, adhesion, and signaling conduction associated GM3 are maintained by GSD $[48,51]$.

\section{Breast cancer}

It has been found that GD3/GD2 synthase ST8SIA1 is over expressed in estrogen receptor (ER)-negative breast tumor, resulting in accumulation of GD2 [52]. Furthermore, accumulation of GD2 can enhance proliferation and tumorigenicity of MDA-MB-231 breast cancer cells by ganglioside-mediated activation of c-Met 
receptor [53], and the detailed mechanism is shown in Figure 3. GD2 has been studied as a breast cancer marker that promotes tumorigenesis [54]. The expression of GSLs in $\mathrm{CD} 44^{\mathrm{hi}} / \mathrm{CD} 24^{\text {lo }}$ breast cancer stem cells (CSCs) and non-CSCs was studied by flow cytometry, and up-regulation of GD2, GD3, GM2, and GD1a in CSCs were detected. Moreover, it was showed that the phenotype can be changed from CSCs to non-CSCs through knockdown ST8SIA1 synthase, and ST8SIA1 synthase can maintain stem cell phenotype in breast CSCs [55]. Interaction of GD3 and GD2 with growth factor receptors also plays a role in maintaining breast CSCs phenotype. In EGFR-positive breast cancer cells, GD3 synthases perhaps involved in gefitinib-resistance [56].

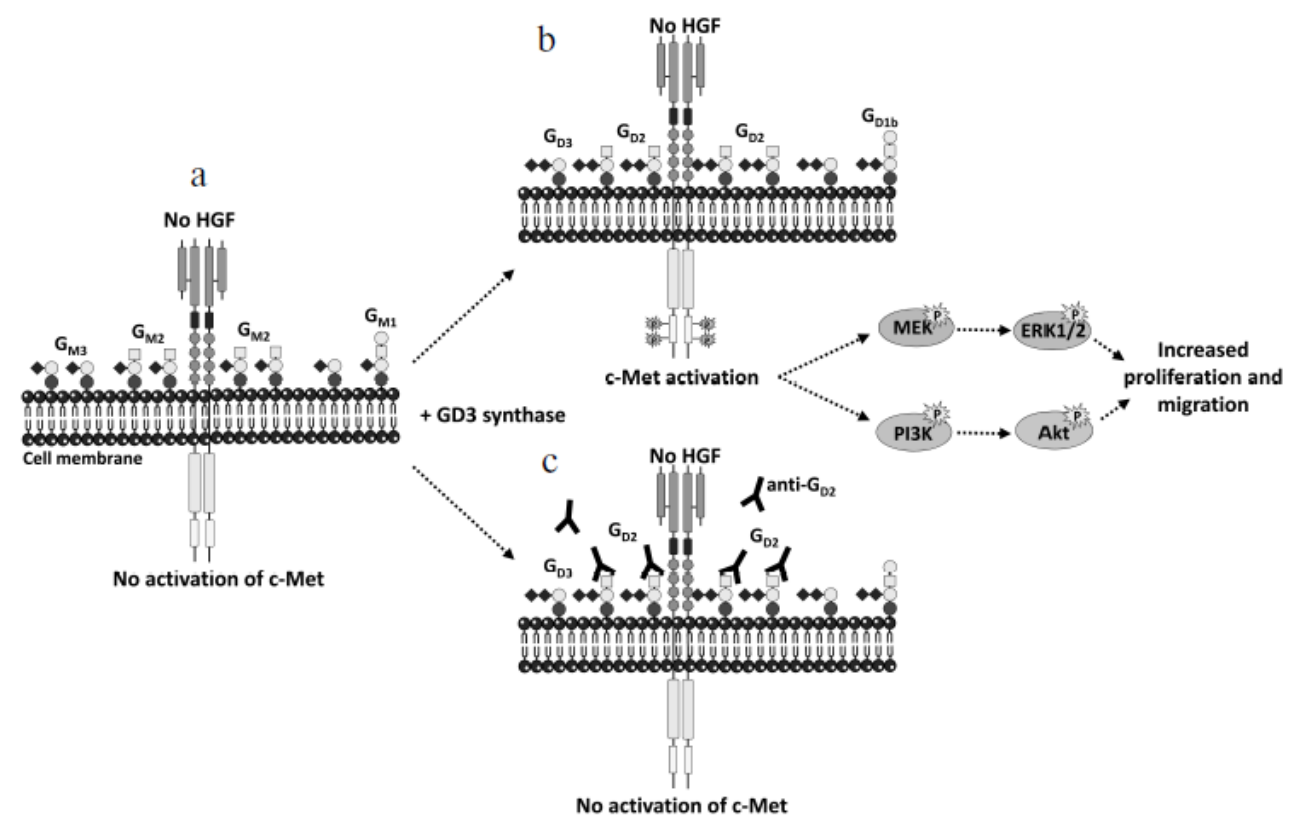

Figure 3. Activation of c-Met by GD2. a) MDA-MB-231 breast cancer cells express mainly GM3 and GM2. b) GD3 synthase expression induces the accumulation of $b$ - and c-series gangliosides, mainly GD2, which can lead to activation of c-Met in the absence of HGF and increase proliferation and migration through the PI3K/Akt and MEK/Erk pathways. c) Anti-GD2 mAb used in competition assays can inhibit c-Met phosphorylation and cells proliferation.

In addition, Gb5 is another potential marker for breast CSCs [57]. Next, exogenous or endogenous expression of GD1b in MCF-7 cell line can result in apoptosis [58]. And over expression of GD1a or its synthase in breast cancer cells can enhance adhesion to brain endothelial cells and reduce interactions with the 
blood-brain barrier, leading to promote metastasis to brain [5,59]. Furthermore, in MCF-7 breast cancer cells, the recognition, clustering and assembly of specific membrane proteins and signal transducers with GSLs in membrane microdomains result in loss of cell-cell adhesion, and subsequently, invasiveness was increased. The loss of cell-cell adhesion was due to sterical hindrance of E-cadherin both localized in and associated with clusters of the GSLs, monosialosyl globopentaosylceramide (MSGb5), and by activation of MSGb5-associated FAK/cSrc signaling complexes and downstream extracellular signal-regulated kinases (ERKs) leading to increased expression and activation of matrix metalloproteinases (MMPs) result in invasion $[60,61]$.

N-glycolyl-GM3 (Neu5Gc GM3) containing N-glycolylneuraminic acid instead of $\mathrm{N}$-acetylneuraminic acid has been detected in many human cancers, such as colon carcinomas and breast cancers, but not in normal human cells [62]. Recent evidences showed that in breast cancer, LacCer, Globo-H, and Fuc-LacCer are significantly increased, and GlcCer and Gb3 are greatly decreased. The cellular effects are mediated by an altered composition of GSLs-enriched microdomains. Importantly, the abnormal Fuc-LacCer is specific to breast cancer. Further studies indicated that fucosyltransferase 1 (FUT1) is as a central player in the biosynthesis of Globo-H and Fuc-LacCer in MDA-MB-453 and MCF-7 cells, and suppressing FUT1 reduces the expression of Globo-H and Fuc-LacCer [63]. The UDP-glucose ceramide glucosyltransferase (UGCG) is also a key enzyme in the synthesis of GSLs. The enhanced proliferation and multidrug resistance in UGCG over expressed breast cancer cells were observed [64].

\section{Lung cancer}

Many studies have demonstrated that GSLs play important roles in lung cancer transformation and progression. $\alpha$-GalCer, which is a specific ligand of invariant natural killer $\mathrm{T}$ (iNKT) cells, shows an inhibitory effect on tumor growth by 
increasing the tumor growth suppressor IFN-r [65]. Besides, it can inhibit inducible nitric oxide synthase (iNOS) expression in lung cancer [66]. In addition, the study showed that $\alpha$-GalCer combined with lipopolysaccharide can obviously promote tumor antigen-specific immune responses and suppress tumor growth [67]. Furthermore, for lung metastasis, host CD40, which is a costimulatory protein, obviously plays an essential role in the effectiveness of $\alpha$-GalCer treatment [68].

It has also been confirmed that increased Gb3 expression not only can result in acquisition of cisplatin resistance, but also reduce GCS-potentiated cisplatin cytotoxicity in non-small cell lung cancer (NSCLC) H1299 cells [69]. In addition, galactocerebrosidase expression that removes galactose from GSLs is reduced in lung cancer [70]. NeuGc-containing gangliosides such as Neu5Gc GM3, are widely expressed in NSCLC, and there are different effects between Neu5Gc GM3 and GM3 on the inhibition of EGFR tyrosine kinase. In addition, GM2 expressed small cell lung cancer (SCLC) shows multiple organ metastases in a severe combined immunodeficient mouse model. However, metastases can be inhibited by treatment with humanized anti-GM2 antibodies, including BIW-8962 and KM8927 [71].

\section{Neural tumor}

The GSLs composition in an F-11 neuroblastoma cell line has been studied, and the results showed that major neutral GSLs are Gb4, Gb3, LacCer, and GlcCer, and major gangliosides are GM3, GD3, O-acetylated GD3, and GD1a, with trace amounts of GD2. Further studies indicated that ganglioside composition alteration, especially GD3 and O-acetylated GD3, is associated with the morphological changes. Other studies have already described a link between certain ganglioside expression and neuroblastomas behavior, and specific gangliosides expression may be as a cancer marker [72,73].

It should be noted that some gliomas contain unusual gangliosides. It has been confirmed that a ratio between GD3 and 9-O-acetylated GD3 on glioblastoma 
promotes tumor survival. Restoring GD3 through removing acetyl group can reduce cancer cells viability by inducing mitochondrial-mediated apoptosis [74,75]. Sulfated glucuronosyl glycosphingolipids (SGGLs) having the Human Natural Killer-1 (HNK-1) epitope are known to be present on a variety of cell adhesion molecules of cancers. Especially for these from neural origin, and regarded as an important biomarker for neural tumors [76]. Further, it has been recognized that there are basically three types of GSL component patterns for gliomas. From several human gliomas, it has been revealed GSL component may reflect subtle differences in cancer growth and state of cells differentiation [77].

Moreover, by adding 1-phenyl-2-decanolyamlno-3-morpholino-1-propanol (PDMP), an effective inhibitor of ceramide glucosyltransferase, the inhibitory effects on GSLs biosynthesis and neurite outgrowth in murine neuroblastoma cell lines were observed [78]. Shedding of membrane gangliosides is characteristic for both human and experimental tumors, and significant ganglioside shedding could influence tumor progression. It has been concluded that shed neuroblastoma gangliosides play an important role in accelerating tumor progression [79].

\section{Leukemia}

In adult T-cell leukemia, glycosyltransferase $\mathrm{FUT}_{7}$ transcription is constitutively activated by Tax protein, leading to sialyl $\mathrm{Le}^{\mathrm{x}}$ synthesis significantly [80]. Caffeic acid phenethyl ester (CAPE) inducing human chronic myelogenous leukemia (CML) K562 cells differentiate to megakaryocytic lineage showed GM3 level was increased [81]. GM3 expression was also upregulated during differentiation of human acute monocytic leukemia THP-1 cells into macrophages [82]. Moreover, it was found that the levels of GM3, lactotriaosylceramide (Lc3) and neolactotetraosylceramide (nLc4) are higher in acute myeloid leukemia (AML) patients than healthy controls, especially the M1 subtype of AML. AML-associated GSLs GM3, Lc3 and nLc4 are possibly involved in initiation and differentiation of AML [83]. In Neu5Gc GM3 expressing 
L1210 mouse lymphocytic leukemia B cells, after silencing of cmah, these cells displayed enhanced NeuAcGM3 expression and an inhibitory effect on anchorage-independent cell growth and tumor progression $[84,85]$.

Three human myeloid leukemia cell lines, including K562, KG1, and HL-60, have been used to study changes in neutral GSLs synthesis with myeloid cell differentiation. The results showed that for K562 and KG1 cells are similar to cells from patients with acute leukemia in expressing two series (globo and neolacto), and for HL-60 cells are similar to mature human myeloid cells in expressing only one series (neolacto). Human myeloid leukemia cells blocked at different stages of differentiation vary in their ability to synthesize neutral GSLs [86]. Other result showed that in primary chronic lymphocytic leukemia (CLL) cells, anti-apoptotic effect from $\alpha$-GlcCer was strongly enhanced by B-cell receptor stimulation [87]. Moreover, in K562 cells, which is a multidrug-resistant variant with GCS and B-cell lymphoma 2 (Bcl-2) co-overexpression, apoptosis was enhanced by adriamycin due to downregulation of Bcl-2 via the ERK pathway, and GCS inhibition also can suppress Bcl-2 [88].

\section{Conclusions and Perspectives}

GSLs are strongly related with tumors, and more and more evidences indicate that they have obvious effects on cancer development and progression. In many types of human cancers, aberrant expression of specific GSLs and related enzymes is associated with tumor progression. Differential expression profiles of GSLs are associated with oncogenic transformation. It is well known that certain GSLs are regarded as tumor-associated antigens. GSLs would become a promising target for cancer therapy.

The expression of GSLs in different cancers is altered, and they can be regarded as biomarkers for some types of cancers or other diseases. Furthermore, external factors can influence the processes that involve in GSLs alterations, such as dietary or 
other lifestyles [89]. Study demonstrated that diets or foods can affect the metabolism-related gene expression of gangliosides resulting in altering ganglioside metabolism as well as ganglioside expression [90]. In addition, some reports also showed that life style-related diseases are strongly associated with aberrant expression of gangliosides [91]. Moreover, molecular pathological epidemiology (MPE) can investigate these external factors. GSL-related biomarkers can be used in MPE study, and GSLs-based MPE study will be a promising direction.

\section{Acknowledgments:}

We thank the China Scholarship Council (CSC) for a Ph.D. fellowship to Changping Zheng. Financial supports from the Centre National de la Recherche Scientifique (CNRS) and the Sorbonne Université in France are gratefully acknowledged.

\section{References:}

[1] Hakomori, S. The glycosynapse. Proc. Natl. Acad. Sci. U.S.A., 2000, 99, 225-232.

[2] Hakomori, S. Structure, organization, and function of glycosphingolipids in membrane. Curr. Opin. Hematol., 2003, 10, 16-24.

[3] Toledo, M.S.; Suzuki, E.; Handa, K.; Hakomori, S. Cell growth regulation through GM3-enriched microdomain (glycosynapse) in human lung embryonal fibroblast WI38 and its oncogenic transformant VA13. J. Bio. Chem., 2004, 279(33), 34655-34664.

[4] Ono, M.; Handa, K.; Sonnino, S.; Withers, D.A.; Nagai, H.; Hakomori, S. GM3 ganglioside inhibits CD9-facilitated haptotactic cell motility: coexpressionof GM3 and CD9 is essential in the downregulation of tumor cell motility and malignancy. Biochem., 2001, 40, 6414-6421.

[5] Zhuo, D.; Li, X.; Guan, F. Biological roles of aberrantly expressed glycosphingolipids and related enzymes in human cancer development and progression. Front. Physiol., 2018, 9, 466.

[6] Daniotti, J.L.; Vilcaes, A.A.; Demichelis, V.T.; Ruggiero, F.M.; Rodriguez-Walker, M. Glycosylation of glycolipids in cancer: basis for development of novel therapeutic approaches. Front. Oncol., 2013, 3, 306.

[7] Wang, H.; Isaji, T.; Satoh, M.; Li, D.; Arai, Y.; Gu, J. Antitumor effects of exogenous ganglioside GM3 on bladder cancer in an orthotopic cancer model. Urolo., 2013, 81, 210 e11-e15.

[8] Ryuji,W.; Chikara, O.; Hiroshi, A.; Toshiko, T.; Makoto, S.; Seiichi, S.; Senji, H.; Atsushi, 
I.; Masaki, S.; Yoichi, A. Ganglioside GM3 overexpression induces apoptosis and reduces malignant potential in murine bladder cancer. Cancer Res., 2002, 62, 3850-3854.

[9] Kawamura, S.; Ohyama, C.; Watanabe, R.; Satoh, M.; Saito, S.; Hoshi, S.; Gasa, S.; Orikasa, S. Glycolipid composition in bladder tumor: a crucial role of GM3 ganglioside in tumor invasion. Int. J. Cancer, 2001, 94(3), 343-347.

[10] Mitsuzuka, K.; Handa, K.; Satoh, M.; Arai, Y.; Hakomori, S. A specific microdomain ("glycosynapse 3") controls phenotypic conversion and reversion of bladder cancer cells through GM3-mediated interaction of $\alpha 3 \beta 1$ integrin with CD9. J. Biol. Chem., 2005, 280, 35545-35553.

[11] Todeschini, A.R.; Dos Santos, J.N.; Handa, K.; Hakomori, S. Ganglioside GM2/GM3 complex affixed on silica nanospheres strongly inhibits cell motility through CD82/cMet-mediated pathway. Proc. Natl. Acad. Sci. U.S.A., 2008, 105(6), 1925-1930.

[12] Ohyama, C. Glycosylation in bladder cancer. Int. J. Clin. Oncol., 2008, 13, 308-313.

[13] Pode, D.; Golijanin, D.; Sherman, Y.; Lebensart, P.; Shapiro, A. Immunostaining of Lewis X in cells from voided urine, cytopathology and ultrasound for noninvasive detection of bladder tumors. J. Urol., 1998, 159. 389-392.

[14] Numahata, K.; Satoh, M.; Handa, K.; Saito, S.; Ohyama, C.; Ito, A.; Takahashi, T.; Hoshi, S.; Orikasa, S.; Hakomori, S. Sialosyl-Le(x) expression defines invasive and metastatic properties of bladder carcinoma. Cancer, 2002, 94, 673-685.

[15] Chatterjee, S.; Alsaeedi, N.; Hou, J.; Ratnam Bandaru, V.V.; Wu, L.; Halushka, M.K.; Pili, R.; Ndikuyeze, G.; Haughey, N.J. Use of a glycolipid inhibitor to ameliorate renal cancer in a mouse model. Plos One, 2013, 8, e63726.

[16] Lin, L.; Huang, Z.; Gao, Y.; Chen, Y.; Hang, W.; Xing, J.; Yan. X. LCMS-based serum metabolic profiling for genitourinary cancer classification and cancer type-specific biomarker discovery. Proteomics, 2012, 12, 2238-2246.

[17] Kawasaki, Y.; Ito, A.; Kakoi, N.; Shimada, S.; Itoh, J.; Mitsuzuka, K.; Arai, Y. Ganglioside, disialosyl globopentaosylceramide (DSGb5), enhances the migration of renal cell carcinoma cells. Tohoku J. Exp. Med., 2015, 236, 1-7.

[18] Saito, S.; Levery. S.B.; Saiyan, M.E.; Goldberg, R.I.; Hakomori, S. Common tetrasaccharide epitope NeuAc $\alpha 2 \rightarrow 3 \mathrm{Gal} \beta 1 \rightarrow 3$ (NeuAc $\alpha 2 \rightarrow 6$ )GalNAc, presented by different carrier glycosylceramides or O-linked peptides, is recognized by different antibodies and ligands having distinct specificities. J. Biol. Chem., 1994, 269, 5644-5652.

[19] Satoh, M.; Handa, K.; Saito, S.; Tokuyama, S.; Ito, A.; Miyao, N.; Orikasa, S.; Hakomori, S. Disialosyl galactosylgloboside as an adhesion molecule expressed on renal cell carcinoma and its relationship to metastatic potential. Cancer Res., 1995, 56. 1932-1938.

[20] Hakomori, S.; Zhang, Y. Glycosphingolipid antigens and cancer therapy. Chem. Biol., 1997, 4, 97-104.

[21] Distler, U.; Souady, J.; Hulsewig, M.; Drmic-Hofman, I.; Haier, J.; Friedrich, A.W.; Karch. H.; Senninger, N.; Dreisewerd, K.; Berkenkamp, S.; Schmidt, M.A.; Peter-Katalinić, J.; Müthing, J. 
Shiga toxin receptor Gb3Cer/CD77: tumor-association and promising therapeutic target in pancreas and colon cancer. Plos One, 2009, 4, e6813.

[22] Falguie res, T.; Maak, M.; von Weyhern, C.; Sarr, M.; Sastre, X.; Poupon, M.F.; Robine, S.; Johannes, L.; Janssen, K.P. Human colorectal tumors and metastases express Gb3 and can be targeted by an intestinal pathogen-based delivery tool. Mol. Cancer Ther., 2008, 7, 2498-2508.

[23] Kovbasnjuk, O.; Mourtazina, R.; Baibakov, B.; Wang, T.; Elowsky, C.; Choti, M.A.; Kane, A.; Donowitz, M. The glycosphingolipid globotriaosylceramide in the metastatic transformation of colon cancer. Proc. Natl. Acad. Sci. U.S.A., 2005, 102(52), 19087-19092.

[24] Kwak, D.H.; Ryu, J.S.; Kim, C.H.; Ko, K.; Ma, J.Y.; Hwang, K.A.; Choo, Y.K. Relationship between ganglioside expression and anti-cancer effects of the monoclonal antibody against epithelial cell adhesion molecule in colon cancer. Exp. Mol. Med., 2011, 43, 693-701.

[25] Yoshioka, K.; Ueno, Y.; Tanaka, S.; Nagai, K.; Onitake, T.; Hanaoka, R.; Watanabe, H.; Chayama, K. Role of natural killer T cells in the mouse colitis-associated colon cancer model. Scand. J. Immunol., 2012, 75, 16-26.

[26] Park, S.Y.; Kwak, C.Y.; Shayman, J.A.; Kim, J.H. Globoside promotes activation of ERK by interaction with the epidermal growth factor receptor. Biochim. Biophys. Acta., 2012, 1820, 1141-1148.

[27] Qu, H.; Liu, J.; Wdzieczak-Bakala, J.; Lu, D.; He, X.; Sun, W.; Sollogoub, M.; Zhang, Y. Synthesis and cytotoxicity assay of four ganglioside GM3 analogues. Eur. J. Med. Chem., 2014, $75,247-257$.

[28] Chung, T.W.; Choi, H.J.; Kim, S.J.; Kwak, C.H.; Song, K.H.; Jin, U.H.; Chang, Y.C.; Chang, H.W.; Lee, Y.C.; Ha, K.T.; Kim, C.H. The ganglioside GM3 is associated with cisplatin-induced apoptosis in human colon cancer cells. Plos One, 2014, 9, e92786.

[29] Shiozaki, K.; Yamaguchi, K.; Sato, I.; Miyagi, T. Plasma membraneassociated sialidase (NEU3) promotes formation of colonic aberrant crypt foci in azoxymethane-treated transgenic mice. Cancer Sci., 2009, 100, 588-594.

[30] Yamaguchi, K.; Shiozaki, K.; Moriya, S.; Koseki, K.; Wada, T.; Tateno, H.; Sato, I.; Asano, M.; Iwakura, Y.; Miyaqi, T. Reduced susceptibility to colitis-associated colon carcinogenesis in mice lacking plasma membrane-associated sialidase. Plos One, 2012, 7, e41132.

[31] Mozzi, A.; Forcella, M.; Riva, A.; Difrancesco, C.; Molinari, F.; Martin, V.; Papini, N.; Bernasconi, B.; Nonnis, S.; Tedeschi, G.; Mazzucchelli, L.; Monti, E.; Fusi, P.; Frattini, M. NEU3 activity enhances EGFR activation without affecting EGFR expression and acts on its sialylation levels. Glycobiology, 2015, 25, 855-868.

[32] Takahashi, K.; Hosono, M.; Sato, I.; Hata, K.; Wada, T.; Yamaguchi, K.; Nitta, K.; Shima, H.; Miyagi, T. Sialidase NEU3 contributes neoplastic potential on colon cancer cells as a key modulator of gangliosides by regulating Wnt signaling. Int. J. Cancer, 2015, 137, 1560-1573.

[33] Livingston, P.O.; Wong, G.Y.; Adluri, S.; Tao, Y.; Padavan, M.; Parente, R.; Hanlon C.; Calves, M.J.; Helling, F.; Ritter, G. Improved survival in stage III melanoma patients with 
GM2 antibodies: a randomized trial of adjuvant vaccination with GM2 ganglioside. J. Clin. Oncol., 1994, 12(5), 1036-1044.

[34] Tai, T.; Cahan, L.D.; Tsuchida, T.; Saxton, R.E.; Irie, R.F.; Morton, D.L. Immunogenicity of melanoma-associated gangliosides in cancer patients. Int. J. Cancer, 1985, 35(5), 607-612.

[35] Gargett, T.; Yu, W.; Dotti, G.; Yvon, E.S.; Christo, S.N.; Hayball, J.D.; Lewis, I.D.; Brenner, M.K.; Brown, M.P. GD2-specific CAR T cells undergo potent activation and deletion following antigen encounter but can be protected from activation-induced cell death by PD-1 Blockade. Mol. Ther., 2016, 24, 1135-1149.

[36] Péguet-Navarro, J.; Sportouch, M.; Popa, I.; Berthier, O.; Schmitt, D.; Portoukalian, J. Gangliosides from human melanoma tumors impair dendritic cell differentiation from monocytes and induce their apoptosis. J. Immunol., 2003, 170(7), 3488-3494.

[37] Tsuchida, T.; Saxton, R.E.; Morton, D.L.; Irie, R.F. Gangliosides of human melanoma. J. Natl. Cancer Inst., 1987, 78, 45-54.

[38] Hersey. P.; Jamal, O.; Henderson, C.; Zardawi, I.; D'Alessandro, G. Expression of the gangliosides GM3, GD3 and GD2 in tissue sections of normal skin, naevi, primary and metastatic melanoma. Int. J. Cancer, 1988, 41, 336-343.

[39] Makino, Y.; Hamamura, K.; Takei, Y.; Bhuiyan, R.H.; Ohkawa, Y.; Ohmi, Y.; Nakashima, H.; Furukawa, K.; Furukawa, K. A therapeutic trial of human melanomas with combined small interfering RNAs targeting adaptor molecules p130Cas and paxillin activated under expression of ganglioside GD3. Biochim. Biophys. Acta., 2016, 1860, 1753-1763.

[40] Furukawa, K.; Kambe, M.; Miyata, M.; Ohkawa, Y.; Tajima, O.; Furukawa, K. Ganglioside GD3 induces convergence and synergism of adhesion and hepatocyte growth factor/Met signals in melanomas. Cancer Sci., 2014, 105, 52-63.

[41] Hamamura, K.; Furukawa, K. Glycosylation is involved in malignant properties of cancer cells. Cancer Transl. Med., 2017, 3(6), 209-213.

[42] Kaneko, K.; Ohkawa, Y.; Hashimoto, N.; Ohmi, Y.; Kotani, N.; Honke, K.; Ogawa, M.; Okajima, T.; Furukawa, K.; Furukawa, K. Neogenin, defined as a GD3-associated molecule by enzyme-mediated activation of radical sources, confers malignant properties via intracytoplasmic domain in melanoma cells. J. Biol. Chem., 2016, 291, 16630-16643.

[43] Cheresh, D.A.; Reisfeld, R.A.; Varki, A.P. O-acetylation of disialoganglioside GD3 by human melanoma cells creates a unique antigenic determinant. Sci., 1984, 225, 844-846.

[44] Kojima, N.; Shiota. M.; Sadahira, Y.; Handa, K.; Hakomori, S. Cell adhesion in a dynamic flow system as compared to static system. J. Bio. Chem., 1992, 267, 17264-17270.

[45] Otsuji, E.; Park, Y.S.; Tashira, K.; Kojima, N.; Toyakuni, T.; Hakomori, S. Inhibition of B16 melanoma metastasis by administration of GM3- or Gg3 lipasomes: blocking adhesion of melanoma cells to endothelia cells (anti-adhesion therapy) via inhibition of GM3-Gg3Cer or GM3-LacCer interaction. Int. J. Oncol., 1995, 6, 319-327.

[46] Chammas, R.; Sonnenburg, J.L.; Watson, N.E.; Tai, T.; Farquhar, M,G.; Varki, N.M.; Varki, A. 
De-N-acetyl-gangliosides in humans: unusual subcellular distribution of a novel tumor antigen. Cancer Res., 1999, 59, 1337-1346.

[47] Liu, J.W.; Sun, P.; Yan, Q.; Paller, A.S.; Gerami, P.; Ho, N.; Vashi, N.; Le Poole, I.C.; Wang, X.Q. De-N-acetyl GM3 promotes melanoma cell migration and invasion through urokinase plasminogen activator receptor signaling-dependent MMP-2 activation. Cancer Res., 2009, 69(22), 8662-8669.

[48] Iwabuchi, K.; Handa, K.; Hakomori, S. Separation of "glycosphingolipid signaling domain" from caveolin-containingmembrane fraction in mouse melanoma B16 cells and its role in celladhesion coupled with signaling. J. Biol. Chem., 1998, 273, 33766-33773.

[49] Iwabuchi, K.; Yamamura, S.; Prinetti, A.; Handa, K.; Hakomori, S. GM3-enriched microdomain involved in cell adhesion and signal transduction through carbohydrate-carbohydrate interaction in mouse melanoma B16 cells. J. Biol. Chem., 1998, 273, 9130-9138.

[50] Iwabuchi, K.; Zhang, Y.; Handa, K.; Withers, D.A.; Sinay, P.; Hakomori, S. Reconstitution of membranes simulating "Glycosignaling Domain" and their susceptibility to Lyso-GM3. J. Biol. Chem., 2000, 275, 15174-15181.

[51] Zhang, Y.; Iwabuchi, K.; Nunomura, S.; Hakomori, S. Effect of synthetic sialyl 2-1 sphingosine and other glycosylsphingosines on the structure and function of the "Glycosphingolipid Signaling Domain (GSD)" in mouse melanoma B16 cells. Biochem., 2000, 39, 2459-2468.

[52] Ruckhaberle, E.; Karn, T.; Rody, A.; Hanker, L.; Gatje, R.; Metzler, D.; Holtrich, U.; Kaufmann, M. Gene expression of ceramide kinase, galactosyl ceramide synthase and ganglioside GD3 synthase is associated with prognosis in breast cancer. J. Cancer Res. Clin. Oncol., 2009, 135, 1005-1013.

[53] Groux-Degroote, S.; Guérardel, Y.; Julien, S.; Delannoy, P. Gangliosides in breast cancer: new perspectives. Biochemistry (Mosc), 2015, 80(7), 808-819.

[54] Battula, V.L.; Shi, Y.; Evans, K.W.; Wang, R.Y.; Spaeth, E.L.; Jacamo, R.O.; Guerra, R.; Sahin, A,A.; Marini, F.C.; Hortobagyi, G.; Mani, S,A.; Andreeff, M. Ganglioside GD2 identifies breast cancer stem cells and promotes tumorigenesis. J. Clin. Invest., 2012, 122, 2066-2078.

[55] Liang, Y.J.; Ding, Y.; Levery, S.B.; Lobaton, M.; Handa, K.; Hakomori, S. Differential expression profiles of glycosphingolipids in human breast cancer stem cells vs. cancer non-stem cells. Proc. Natl. Acad. Sci. U.S.A., 2013, 110, 4968-4973.

[56] Liang, Y.J.; Wang, C.Y.; Wang, I.A.; Chen, Y.W.; Li, L.T.; Lin, C.Y.; Ho M.Y.; Chou, T.L.; Wang, Y.H.; Chiou, S.P.; Lin, Y.J.; Yu, J. Interaction of glycosphingolipids GD3 and GD2 with growth factor receptors maintains breast cancer stem cell phenotype. Oncotarget, 2017, 8, 47454-47473.

[57] Cheung, S.K.; Chuang, P.K.; Huang, H.W.; Hwang-Verslues, W.W.; Cho, C.H.; Yang, W.B.; Shen, C.N.; Hsiao, M.; Hsu, T.L.; Chang, C.F.; Wong, C.H. Stage-specific embryonic antigen-3 (SSEA-3) and b3GalT5 are cancer specific and significant markers for breast cancer stem cells. 
Proc. Natl. Acad. Sci. U.S.A., 2016, 113, 960-965.

[58] Ha, S.H.; Lee, J.M.; Kwon, K.M.; Kwak, C.H.; Abekura, F.; Park, J.Y.; Cho, S.H.; Lee, K.; Chang, Y.C.; Lee, Y.C.; Choi, H.J.; Chung, T.W.; Ha, K.T.; Chang, H.W.; Kim, C.H. Exogenous and endogeneous disialosyl ganglioside GD1b induces apoptosis of MCF-7 human breast cancer cells. Int. J. Mol. Sci., 2016, 17, E652.

[59] Drolez, A.; Vandenhaute, E.; Delannoy, C.P.; Dewald, J.H.; Gosselet, F.; Cecchelli, R.; Julien, S.; Dehouck, M.P.; Delannoy, P.; Mysiorek, C. ST6GALNAC5 expression decreases the interactions between breast cancer cells and the human blood-brain barrier. Int. J. Mol. Sci., 2016, 17, E1309.

[60] Van Slambrouck, S.; Hilkens, J.; Steelant, W.F. Ether lipid 1-O-octadecyl-2-O-methyl-3-glycero-phosphocholine inhibits cell-cell adhesion through translocation and clustering of E-cadherin and episialin in membrane microdomains. Oncol. Rep., 2008, 19(1), 123-128.

[61] Van Slambrouck, S.; Steelant, W.F. Clustering of monosialyl-Gb5 initiates downstream signalling events leading to invasion of MCF-7 breast cancer cells. Biochem. J., 2007, 401(3), 689-699.

[62] Malykh, Y.N.; Schauer, R.; Shaw, L. N-Glycolylneuraminic acid in human tumours. Biochimie, 2001, 83, 623-634.

[63] Zhu, T.; Xu, L.; Xu, X.; Wang, Z.; Zhu, J.; Xie, Q.; Zhang, B.; Wang, Y.; Ju, L.; He, Y.; Ye, X.; Zhou, D.; Li, Y. Analysis of breast cancer-associated glycosphingolipids using electroXspray ionization-linear ion trap quadrupole mass spectrometry. Carbohydr, Res., 2015, 402, 189-199.

[64] Wegner, M.S.; Schomel, N.; Gruber, L.; Ortel, S.B.; Kjellberg, M.A.; Mattjus, P.; Kurz, J.; Trautmann, S.; Peng, B.; Wegner, M.; Kaulich, M.; Ahrends, R.; Geisslinger, G.; Sabine, S. UDP- glucose ceramide glucosyltransferase activates AKT, promoted proliferation, and doxorubicin resistance in breast cancer cells. Cell. Mol. Life Sci., 2018, 75, 3393-3410.

[65] Hasegawa, H.; Yamashita, K.; Otubo, D.; Fujii, S.; Kamigaki, T.; Kuroda, D.; Kakeji, Y. Allogeneic DCG promote lung NK cell activation and antitumor effect after invariant NKT cell activation. Anticancer Res., 2014, 34, 3411-3417.

[66] Ito, H.; Ando, T.; Seishima, M. Inhibition of iNOS activity enhances the anti-tumor effects of a-galactosylceramide in established murine cancer model. Oncotarget, 2015, 6, 41863-41874.

[67] Ando, T.; Ito, H.; Arioka, Y.; Ogiso, H.; Seishima, M. Combination therapy with a-galactosylceramide and a Toll-like receptor agonist exerts an augmented suppressive effect on lung tumor metastasis in a mouse model. Oncol. Rep., 2015, 33(2), 826-32.

[68] Yamashita, K.; Hasegawa, H.; Fujita, M.; Nishi, M.; Tanaka, T.; Arimoto, A.; Suzuki, S.; Kamiqaki, T.; Kakeji, Y. Host CD40 is essential for DCG treatment against metastatic lung cancer. Anticancer Res., 2016, 36, 3659-3665.

[69] Tyler, A.; Johansson, A.; Karlsson, T.; Gudey, S.K.; Brannstrom, T.; Grankvist, K.; Behnam-Motlaqh, P. Targeting glucosylceramide synthase induction of cell surface 
globotriaosylceramide (Gb3) in acquired cisplatin-resistance of lung cancer and malignant pleural mesothelioma cells. Exp. Cell Res., 2015, 336, 23-32.

[70] Peng, J.; Chen, B.; Shen, Z.; Deng, H.; Liu, D.; Xie, X.; Gan, X.; Xu, X.; Huang, Z.; Chen, J. DNA promoter hypermethylation contributes to down-regulation of galactocerebrosidase gene in lung and head and neck cancers. Int. J. Clin. Exp. Pathol., 2015, 8, 11042-11050.

[71] Yamada, T.; Bando, H.; Takeuchi, S.; Kita, K.; Li, Q.; Wang, W.; Akinaqa, S.; Nishioka, Y.; Sone, S.; Yano, S. Genetically engineered humanized anti-ganglioside GM2 antibody against multiple organ metastasis produced by GM2-expressing small-cell lung cancer cells. Cancer Sci., 2011, 102, 2157-2163.

[72] Ariga, T.; Blaine, G.M.; Yoshino, H.; Dawson, G.; Kanda, T.; Zeng, G.C.; Kasama, T.; Kushi, Y.; Yu, R.K. Glycosphingolipid Composition of murine neuroblastoma cells: O-acetylesterase gene downregulates the expression of O-acetylated GD3. Biochem., 1995, 34, 11500-11507.

[73] Kaneko, T.; Okita, H.; Nakajima, H.; Iijima, K.; Ogasawara, N.; Miyagawa, Y.; Katagiri, Y.U.; Nakagawa, A.; Kiyokawa, N.; Sato, T.; Fujimoto, J. Neuroblastoma cells can be classified according to glycosphingolipid expression profiles identified by liquid chromatography-tandem mass spectrometry. Int. J. Oncol., 2010, 37(5), 1279-1288.

[74] Yeh, S.C.; Wang, P.Y.; Lou, Y.W.; Khoo, K.H.; Hsiao, M.; Hsu, T.L.; Wong, C.H. Glycolipid GD3 and GD3 synthase are key drivers for glioblastoma stem cells and tumorigenicity. Proc. Natl. Acad. Sci. U.S.A., 2016, 113(20), 5592-5597.

[75] Birks, S.M.; Danquah, J.O.; King, L.; Vlasak, R.; Gorecki, D.C.; Pilkington, G.J. Targeting the GD3 acetylation pathway selectively induces apoptosis in glioblastoma. Neuro. Oncol., 2011, 13, 950-960.

[76] Ariga, T.; Suetake, K.; Nakane, M.; Kubota, M.; Usuki, S.; Kawashima, I.; Yu, R.K. Glycosphingolipid antigens in neural tumor cell lines and anti-glycosphingolipid antibodies in sera of patients with neural tumors. Neurosignals, 2008, 16, 226-234.

[77] Jennemann, R.; Rodden, A.; Bauer, B.L.; Mennel, H.D.; Wiegandt, H. Glycosphingolipids of human gliomas. Cancer Res., 1990, 50(23), 7444-7449.

[78] Uemura, K.; Sugiyama, E.; Taketomi, T. Effects of an inhibitor of glucosylceramide synthase on glycosphingolipid synthesis and neurite outgrowth in murine neuroblastoma cell lines. Biochem. J., 1991, 110 (1), 96-102.

[79] Li, R.X.; Ladisch, S. Shedding of human neuroblastoma gangliosides. Biochim. Biophys. Acta., 1991, 1083, 57-64.

[80] Hiraiwa, N.; Hiraiwa, M.; Kannagi, R. Human T-cell leukemia virus-1 encoded Tax protein transactivates alpha 1-->3 fucosyltransferase Fuc-T VII, which synthesizes sialyl Lewis X, a selectin ligand expressed on adult T-cell leukemia cells. Biochem. Biophys. Res. Commun., 1997, 231(1), 183-186.

[81] Jin, U.H.; Chung, T.W.; Song, K.H.; Kwak, C.H.; Choi, H.J.; Ha, K.T.; Chang, Y.C.; Lee, Y.C.; Kim, C.H. Ganglioside GM3 is required for caffeic acid phenethyl ester-induced 
megakaryocytic differentiation of human chronic myelogenousleukemia K562 cells. Biochem. Cell Biol., 2014, 92, 243-249.

[82] Delannoy, C.P.; Rombouts, Y.; Groux-Degroote, S.; Holst, S.; Coddeville, B.; Harduin-Lepers, A.; Wuhrer, M.; Elass-Rochard, E.; Guérardel, Y. Glycosylation changes triggered by thedifferentiation of monocytic THP-1 cell line into macrophages. J. Proteome Res., 2017, 16, 156-169.

[83] Wang, Z.; Wen, L.; Ma, X.; Chen, Z.; Yu, Y.; Zhu, J.; Wang, Y.; Liu, Z.; Liu, H.; Wu, D.; Zhou, D.; Li, Y. High expression of lactotriaosylceramide, a differentiation-associated glycosphingolipid, in the bone marrow of acute myeloid leukemia patients. Glycobiology, 2012, 22, 930-938.

[84] Fernandez-Marrero, Y.; Hernandez, T.; Roque-Navarro, L.; Talavera, A.; Moreno, E.; Grinan, T.; Vázquez, A.M.; de Acosta, C.M.; Pérez, R.; López-Requena, A. Switching on cytotoxicity by a single mutation at the heavy chain variable region of an anti-ganglioside antibody. Mol. Immunol., 2011, 48, 1059-1067.

[85] Casadesus, A.V.; Fernandez-Marrero, Y.; Clavell, M.; Gomez, J.A.; Hernandez, T.; Moreno, E.; López-Requena, A. A shift from N-glycolyl- to N-acetyl-sialic acid in the GM3 ganglioside impairs tumor development in mouse lymphocytic leukemia cells. Glycoconj. J., 2013, 30, 687-699.

[86] Buehler, J.; Qwan, E.; De Gregorio, M.W.; Macher, B.A. Biosynthesis of glycosphingolipids by human myeloid leukemia cells. Biochem., 1985, 24(24), 6978-84.

[87] Schwamb, J.; Feldhaus, V.; Baumann, M.; Patz, M.; Brodesser, S.; Brinker, R.; Claasen, J.; Pallasch, C.P.; Hallek, M.; Wendtner, C.M.; Frenzel, L.P. B-cell receptor triggers drug sensitivity of primary CLL cells by controlling glucosylation of ceramides. Blood, 2012, 120, 3978-3985.

[88] Wang, Q.; Zou, J.; Zhang, X.; Mu, H.; Yin, Y.; Xie, P. Glucosylceramide synthase promotes Bcl-2 expression via the ERK signaling pathway in the K562/A02 leukemia drug-resistant cell line. Int. J. Hematol., 2014, 100, 559-566.

[89] Okuda, T. Dietary control of ganglioside expression in mammalian tissues. Int. J. Mol. Sci., 2020, $21,177$.

[90] Okuda, T. A low-carbohydrate ketogenic diet promotes ganglioside synthesis via the transcriptional regulation of ganglioside metabolism-related genes. Sci. Rep., 2019, 9, 7627.

[91] Inokuchi J. Physiopathological function of hematoside (GM3 ganglioside). Proc. Jpn. Acad. Ser. B Phys. Biol. Sci., 2011, 87(4), 179-198. 